\title{
Ramucirumab: hitting another target in gastric cancer
}

This article was published in the following Dove Press journal:

Gastrointestinal Cancer: Targets and Therapy

24 February 2015

Number of times this article has been viewed

\author{
Melissa J Cohen' \\ Zev A Wainberg 2,3 \\ 'Division of Hematology and \\ Oncology, University of California \\ Los Angeles (UCLA), Porter Ranch, \\ ${ }^{2}$ School of Medicine, ${ }^{3}$ Gastrointestinal \\ Oncology Program, University of \\ California Los Angeles (UCLA), \\ Los Angeles, CA, USA
}

\begin{abstract}
Gastric cancer remains a lethal disease worldwide. Despite a recent increase in available systemic therapeutics, the prognosis of patients with advanced disease remains poor. A number of targeted therapies have been evaluated in gastric cancer, albeit with mixed results. Trastuzumab - the monoclonal antibody blocking HER2 - has contributed to improved survival for a minority of gastric cancer patients. Ramucirumab - a VEGF2 inhibitor - blocks the proliferation and migration of endothelial cells. The US Food and Drug Administration has recently approved ramucirumab (Cyramza) to treat patients with advanced or metastatic gastric cancer in the second-line setting following progression on fluoropyrimidine- or platinum-containing therapy. This approval was granted on the basis of the REGARD trial that demonstrated that patients treated with ramucirumab experienced a median overall survival of 5.2 months compared with 3.8 months in patients receiving placebo. Results from the RAINBOW randomized Phase III clinical trial have also shown improvements in overall survival for ramucirumab plus paclitaxel versus paclitaxel alone in patients previously treated for locally advanced or metastatic gastric cancer. In both studies, the most common adverse events reported were hypertension, diarrhea, and fatigue. Because of the encouraging advancement in novel targeted therapies, the future is looking brighter for patients with advanced gastric cancer. While this research is promising, further evaluation and identification of targets or population subsets that can be identified prospectively will likely help predict who will benefit most from these therapies.
\end{abstract}

Keywords: metastatic gastric cancer, ramucirumab, targeted therapies, angiogenesis, Cyramza

\section{Introduction}

Gastric cancer is the second most common cause of cancer death globally. In 2014, it has been estimated there will be 22,240 cases diagnosed and 10,990 deaths from gastric cancer. Among the major types of malignancies, gastric cancer is the 14 th most common in the US. ${ }^{1}$ Though gastric cancer is more prevalent in countries such as Japan and the People's Republic of China, it remains an equally lethal disease regardless of ethnicity. Overall 5-year survival estimates range from $57 \%$ for early-stage disease to $4 \%$ for distant metastatic disease. Unfortunately, more than $80 \%$ of patients present with either locally advanced or metastatic disease. ${ }^{2-4}$ Some risk factors for gastric cancer have been identified and include: Helicobacter pylori infection, chronic atrophic gastritis, diets low in fruits and vegetables and rich in salted, smoked, or preserved foods, cigarette smoking, and alcohol abuse, among others. ${ }^{5}$

Treatment of gastric cancer is guided by the size and location of the tumor, as well as the stage of disease at diagnosis. For the group of patients who are diagnosed with
Correspondence: Melissa J Cohen Division of Hematology and Oncology, UCLA, 19950 Rinaldi St, Suite 310, Porter Ranch, CA 91326, USA

$\mathrm{Tel}+\mid$ 8I827| 2500

Email mjcohen@mednet.ucla.edu
Gastrointestinal Cancer: Targets and Therapy 2015:5 73-77 73

Dovepress

(c) (i) (8) $\odot 215$ Cohen and Wainberg. This work is published by Dove Medical Press Limited, and licensed under Creative Commons Attribution - Non Commercial (unported, v3.0) $\mathrm{BY}$ NC License. The full terms of the License are available at http://creativecommons.org/licenses/by-n/3.0/. Non-commercial uses of the work are permitted without any further
permission from Dove Medical Press Limited, provided the work is properly attributed. Permissions beyond the scope of the License are administered by Dove Medical Press Limited. Information on permission from Dove Medical Press $L$ Limited, provided the work is properly attributed. P
how to request permission may be found at: http://www.dovepress.com/permissions.php 
early-stage disease, surgery by means of gastrectomy can be curative. For patients with locoregional disease, treatment usually involves a multidisciplinary approach using chemotherapy in conjunction with surgery. For the majority of patients diagnosed with advanced disease, combination chemotherapy has been the rule for the past two decades, with recent modest improvement in outcomes. In the firstline setting, combination chemotherapy produces response rates up to $54 \%$; despite this, median survival remains a mere 9-10 months. ${ }^{6,7}$ Although various chemotherapeutic agents alone or in combination have been studied and continue to be explored, the main active drugs include 5-fluorouracil, cisplatin, oxaliplatin, irinotecan, docetaxel, and paclitaxel. Historically, patients whose cancer progresses following firstline therapy have a poor overall prognosis with median overall survival of 3.5 months with best supportive measures. ${ }^{8}$

Until recently, research in gastric cancer has lagged behind that of other malignancies.

The concept of targeted therapy has only gained momentum in the treatment of advanced gastric cancer over the past 5 years. As is seen in breast cancers, amplification of the tyrosine kinase receptor HER2 has been found to be present in gastric cancer. ${ }^{9}$ This cell surface receptor when bound to a ligand initiates a signal transduction cascade that leads toward proliferation, adhesion, migration, and differentiation. Historically, HER2-amplified gastric cancer has been felt to have a worse prognosis, with median survival averaging 5.5 months compared with 12.6 months in nonamplified patients. ${ }^{10-12}$ In 2010, results from the ToGA trial demonstrated that the addition of trastuzumab (Herceptin) to chemotherapy improved survival by almost 3 months in patients with HER2-positive disease. Unfortunately, only $10 \%-15 \%$ of patients with advanced gastric cancer have HER2-positive disease. ${ }^{13}$

\section{Targeting angiogenesis in cancer therapy}

Abnormal angiogenesis is thought to be essential to a tumor's ability to grow and spread; therefore, antiangiogenic strategies have been exploited as possible cancer treatments. One of the main drivers of angiogenesis is the interaction between VEGF and its cell surface receptors (Figure 1). Increased expression of VEGF in tumor and serum has been associated with poor prognosis in gastric cancer. ${ }^{14-16}$ Bevacizumab is a humanized IgG1 monoclonal antibody against VEGF. It selectively binds to circulating VEGF, inhibiting its binding to the

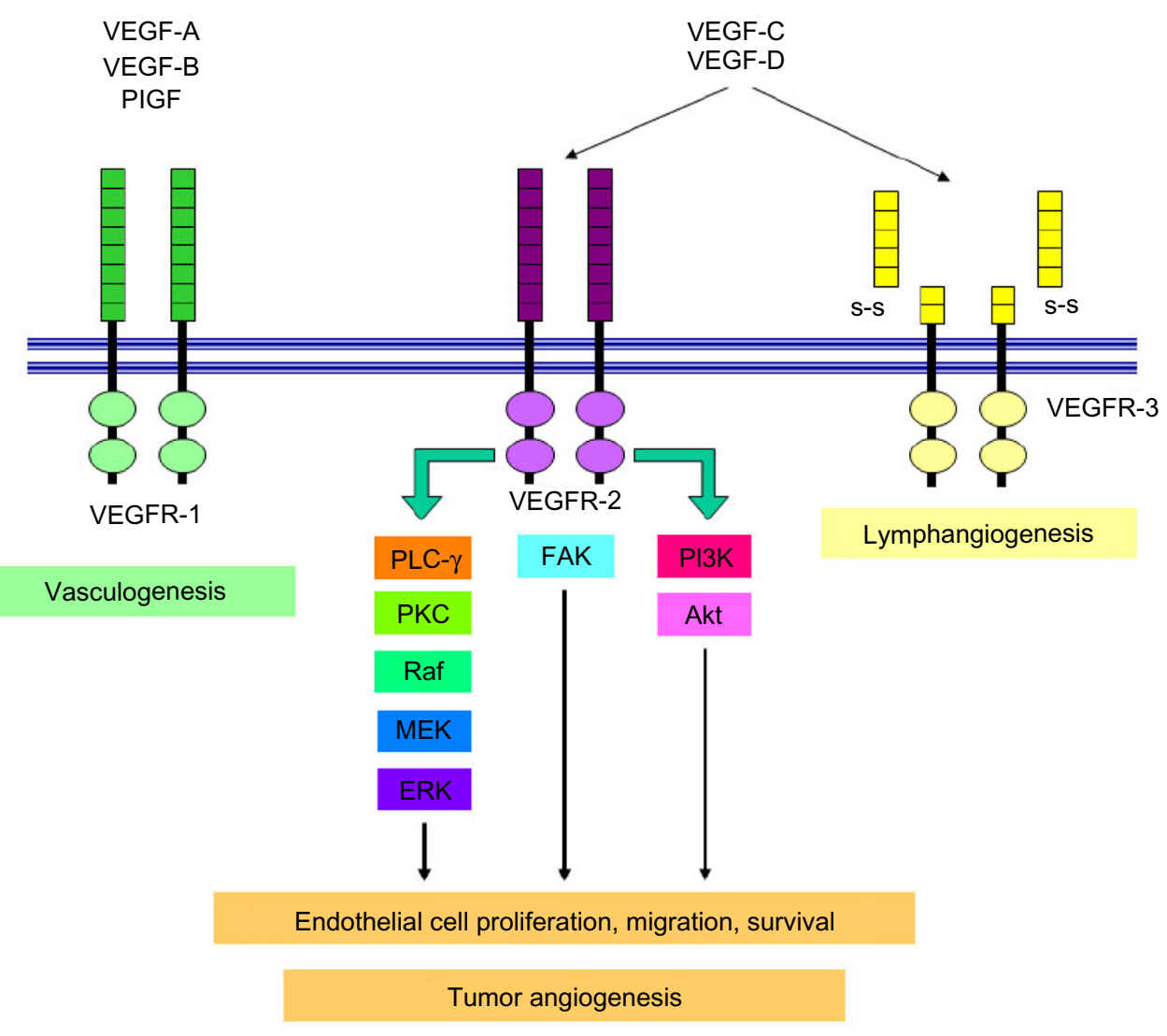

Figure I VEGF pathway. 
corresponding receptors and hence inhibiting angiogenesis. Though results from Phase II trials with bevacizumab in gastric cancer seemed encouraging, the pivotal Phase III study failed to meet its primary endpoint of overall survival. ${ }^{17,18}$ In the AVAGAST study, investigators examined the efficacy of bevacizumab in over 700 patients with advanced gastric cancer who were randomized to capecitabine and cisplatin plus bevacizumab or capecitabine and cisplatin plus placebo. The median overall survival was 12.1 months with chemotherapy plus bevacizumab versus 10.1 months with chemotherapy plus placebo. Though not statistically significant, there appeared to be a trend toward improved survival in the bevacizumab arm. ${ }^{18}$ Interestingly, in retrospective subset analysis, when divided into subgroups based upon geographical location, there was a considerable benefit in patients from Europe and the US but not in patients from Asia. ${ }^{19}$ Similarly, the AVATAR study conducted in the People's Republic of China failed to improve outcomes in patients with advanced gastric cancer when bevacizumab was added to a capecitabine-cisplatin backbone. There was no difference in overall survival between the two arms and progression-free survival was similar. ${ }^{20}$

Given a possible signal of activity in these trials, ramucirumab was investigated as an alternative antiangiogenic strategy. Ramucirumab is a fully human receptor targeted monoclonal antibody that blocks VEGFR-2 and inhibits downstream signaling in the pathway involved in the formation and maintenance of blood vessels that supply blood to tumors. The mechanism is distinct from that of bevacizumab in that it binds to the receptor directly, rather than with bevacizumab where it is binding to the ligand (VEGF-A). ${ }^{20}$

\section{Ramucirumab}

Preclinical evaluations of antibodies blocking various VEGF receptors were performed with DC-101, an antibody directed against murine VEGFR-2. ${ }^{21}$ In these mouse models, inhibition of metastases was demonstrated. A number of Phase I studies of ramucirumab have been completed and established a maximum tolerated dose of $13 \mathrm{mg} / \mathrm{kg}$ weekly. Dose limiting toxicities included grade 3 hypertension and deep vein thrombosis. Efficacy results from these studies showed a signal for single-agent activity with responses seen in gastric cancer. ${ }^{22}$ Because of the signal seen in gastric cancer, ramucirumab was tested in Phase II/III clinical trials focusing on advanced gastric cancer in the second-line setting.

\section{REGARD study}

The pivotal REGARD study was published in October 2013 and its results led to the Food and Drug Administration granting priority review status to ramucirumab. The work by Fuchs et al showed ramucirumab to be the first biologic treatment given as a single agent to increase overall survival in patients with advanced gastric cancer who progress after front-line therapy. Their findings validate VEGF-2 signaling as an important target. This multinational Phase III double-blind study contained over 350 patients with advanced gastric cancer/gastroesophageal junction adenocarcinoma who were randomly assigned in a 2:1 ratio to receive best supportive care plus either ramucirumab $8 \mathrm{mg} / \mathrm{kg}$ or placebo once every 2 weeks intravenously. Median duration of treatment was 8 weeks in the ramucirumab group and 6 weeks in the placebo group. Patients treated with single-agent ramucirumab achieved a median overall survival of 5.2 months compared to 3.8 months for patients in the placebo arm $(n=117)$ and the overall survival hazard ratio was $0.776(P=0.0473)$. This corresponded to a $22 \%$ reduction in death rate (Table 1 ). Patients in the ramucirumab arm achieved a median progression-free survival of 2.1 months compared to 1.3 months for those in the placebo arm. The progression-free survival hazard ratio was $0.483(P<0.0001)$. Patients also appeared to tolerate the VEGFR-2 inhibitor very well; the most common $(>5 \%$ incidence) grade $>3$ adverse events were hypertension and abdominal pain. ${ }^{23}$

Though a numerical benefit of median survival of 22 days is modest, these results are equivalent to those seen with chemotherapy alone in the second line. For instance, COUGAR-02 - a second-line study which evaluated the benefit of docetaxel chemotherapy plus best supportive care versus best supportive care alone - showed an overall

Table I Phase III studies of ramucirumab for gastric cancer

\begin{tabular}{|c|c|c|c|c|c|}
\hline Trial & Number of patients & Randomization arms & Response rate & Progression-free survival & Overall survival \\
\hline REGARD & $355(2: 1)$ & Arm I: BSC $+\mathrm{R}$ & $3 \%$ & 2.1 months & 5.2 months \\
\hline \multirow[t]{2}{*}{ (NCT009/7384) } & Arm I: 238 & Arm 2: BSC $+\mathrm{P}$ & $3 \%$ & 1.3 months & 3.8 months \\
\hline & Arm 2: 117 & & & $(\mathrm{HR} 0.48 ; P<0.000 \mathrm{I})$ & (HR 0.7; $P=0.047)$ \\
\hline RAINBOW & $665(1: 1)$ & Arm I: PAC + R & $28 \%$ & 4.4 months & 9.6 months \\
\hline \multirow[t]{2}{*}{ (NCT0II70663) } & Arm I: 330 & Arm 2: $P A C+P$ & $16 \%$ & 2.8 months & 7.3 months \\
\hline & Arm 2: 335 & & $P<0.0001$ & $(\mathrm{HR} 0.63 ; P<0.000 \mathrm{I})$ & (HR 0.8; $P=0.016)$ \\
\hline
\end{tabular}

Abbreviations: BSC, best supportive care; HR, hazard ratio; P, placebo; PAC, paclitaxel; R, ramucirumab. 
survival benefit of 1.6 months. In this study, over 150 patients were randomized in a 1:1 fashion to receive docetaxel at $75 \mathrm{mg} / \mathrm{m}^{2}$ every 21 days plus best supportive care versus best supportive care alone. Patients who received docetaxel survived 5.2 months versus 3.6 months for best supportive care alone (HR $0.67 ; P=0.01$ ). In addition to a $33 \%$ reduction in death, patients in the docetaxel arm reported less pain, nausea and vomiting, abdominal pain, and constipation. Neutropenia and fever were the main toxicities. ${ }^{24}$

\section{RAINBOW study}

Given its efficacy and favorable side effect profile, studies were conducted adding ramucirumab to chemotherapy in hopes there would be synergism. According to the randomized Phase III RAINBOW study, the combination of ramucirumab and paclitaxel resulted in a significant prolongation in survival compared to single-agent paclitaxel for the second-line treatment of patients with metastatic gastric cancer. The study also showed that the response rate was increased with the combination. In addition, significant gains in quality of life for the combination of ramucirumab and paclitaxel over paclitaxel alone were demonstrated. ${ }^{25}$ This trial set out to compare the safety and efficacy of paclitaxel with or without ramucirumab in patients with locally advanced gastric cancer of the stomach or gastroesophageal junction, refractory or progressed after first-line chemotherapy. Over 650 patients from 200 study centers in 30 countries were enrolled. Patients were randomized in a 1:1 fashion to receive ramucirumab plus paclitaxel $(n=300)$ or placebo plus paclitaxel $(n=355)$. The ramucirumab and paclitaxel combination achieved its primary endpoint of overall survival by $>2$ months when compared to paclitaxel alone. In addition, patients had a near doubling of the secondary endpoints: progression-free survival and overall response rate with the combination (Table 1). The most common adverse events (grade $>3$ ) occurring in the ramucirumab plus paclitaxel arm included neutropenia, leucopenia, hypertension, fatigue, and abdominal pain. Grade $\geq 3$ neutropenia occurred in $40 \%$ of patients in the combination arm compared to $18.8 \%$ of patients in the paclitaxel alone arm. Though the incidence of neutropenia was greater in the combination arm, febrile neutropenia rates were relatively comparable to single-agent paclitaxel $(3.1 \%$ versus $2.4 \%$, respectively).

\section{Conclusion}

Ramucirumab is the first targeted agent to demonstrate single-agent activity in metastatic gastric cancer and it is also the first antiangiogenic therapy to show a survival benefit in this disease. Studies in several other settings are ongoing in metastatic gastric cancer. The success of ramucirumab in these recent studies is promising, but there remains a critical need to identify predictive molecular markers to select the patients who might benefit most from such targeted therapy.

A major challenge for antiangiogenic therapies is the lack of a robust biomarker that can guide selection of patients for whom the therapy is most likely to be beneficial. Attempts to do this for bevacizumab in colon and lung cancer have either been inconclusive or not validated. In the AVAGAST trial, high circulating VEGF-A levels and/or low neuropilin levels were associated with a benefit in post hoc analysis. ${ }^{26}$ For ramucirumab, such targets have not been identified. To date, in both large pivotal studies, there have been no data presented that identifies whether the HER2-positive subgroup benefits in the same manner as the HER2-negative subgroup.

Recently, researchers from The Cancer Genome Atlas Network project have found that stomach cancers fall into four distinct molecular subtypes: Epstein-Barr virus positive, microsatellite instable, genomically stable, and those with alterations that suggest genomic instability. ${ }^{27}$ Perhaps it is discoveries such as this that can help better determine which targeted therapies are appropriate for which types of cancer and each nationality.

\section{Disclosure}

The authors report no conflicts of interest in this work.

\section{References}

1. Globocan 2008 Cancer Fact Sheet: stomach cancer incidence and mortality worldwide in 2008 [webpage on the Internet]. Available from: http:// globocan.iarc.fr/old/FactSheets/cancers/stomach-new.asp. Accessed October 28, 2014.

2. Survival rates for stomach cancer, by stage. Atlanta, GA: American Cancer Society; 2014 [updated May 27, 2014]. Available from: http:// www.cancer.org/cancer/stomachcancer/detailedguide/stomach-cancersurvival-rates. Accessed September 12, 2014.

3. Thompson GB, Van Heerden JA, Starr MG. Adenocarcinoma of the stomach: are we making progress? Lancet. 1993;342(8873):713-718.

4. Jemal A, Bray F, Center MM, Ferlay J, Ward E, Forman D. Global cancer statistics. CA Cancer J Clin. 2011;61(2):69-90.

5. Bornschein J, Rokkas T, Selgrad M, Malfertheiner P. Gastric cancer: clinical aspects, epidemiology, and molecular background. 2011; 16(Suppl 1):45-52.

6. Wagner AD, Unverzagt S, Grothe W, et al. Chemotherapy for advanced gastric cancer. Cochrane Database Syst Rev. 2010;3:CD004064.

7. Cunningham D, Starling N, Rao S, et al. Capecitabine and oxaliplatin for advanced esophagogastric cancer. N Engl J Med. 2008;358(1): 36-46.

8. Kim HS, Kim HJ, Kim SY, et al. Second-line chemotherapy versus supportive cancer treatment in advanced gastric cancer: a meta-analysis. Ann Oncol. 2013;24(11):2850-2854.

9. Gravalos C, Jimeno A. HER2 in gastric cancer: a new prognostic factor and a novel therapeutic target. Ann Oncol. 2008;19(9):1523-1529. 
10. Jorgensen JT, Hersom M. HER2 as a prognostic marker in gastric cancer: a systematic analysis of data from the literature. $J$ Cancer. 2012;3:137-144.

11. Bang YJ, Van Cutsem E, Feyereislova A, et al. Trastuzumab in combination with chemotherapy versus chemotherapy alone for treatment of HER2-positive advanced gastric or gastro-oesophageal junction cancer (ToGA): a Phase III, open-label, randomised controlled trial. Lancet. 2010;376(9742):687-697.

12. Chua TC, Merrett ND. Clinicopathologic factors associated with HER2-positive gastric cancer and its impact on survival outcomes: a systematic review. Int J Cancer. 2012;130(12):2845-2856.

13. Ferrara N, Gerber HP, LeCouter J. The biology of VEGF and its receptors. Nat Med. 2003;9(6):669-676

14. Zhao ZQ, Yang S, Lu HS. Expression of midkine and vascular endothelial growth factor in gastric cancer and the association of high levels with poor prognosis and survival. Mol Med Rep. 2012;5(2):415-419.

15. Cabuk D, Basaran G, Celikel C, et al. Vascular endothelial growth factor, hypoxia-inducible factor $1 \alpha$ and CD34 expressions in early-stage gastric tumors: relationship with pathological factors and prognostic impact on survival. Oncology. 2007;72(1-2):111-117.

16. Juttner S, Wissmann C, Jons $\mathrm{T}$, et al. Vascular endothelial growth factor-D and its receptor VEGFR-3: two novel independent prognostic markers in gastric adenocarcinoma. J Clin Oncol. 2006;24(2):228-240.

17. Shah MA, Ramanathan RK, Ilson DH, et al. Multicenter Phase II study of irinotecan, cisplatin, and bevacizumab in patients with metastatic gastric or gastroesophageal junction adenocarcinoma. J Clin Oncol. 2006;24(33):5201-5206.

18. Ohtsu A, Shah MA, Van Cutsem E, et al. Bevacizumab in combination with chemotherapy as first-line therapy in advanced gastric cancer: a randomized, double-blind, placebo-controlled Phase III study. J Clin Oncol. 2011;29(30):3968-3976.

19. Shah M, Van Cutsem E, Kang YK, et al. Survival analysis according to disease subtype in AVAGAST: first-line capecitabine and cisplatin plus bevacizumab (bev) or placebo in patients (pts) with advanced gastric cancer [abstract]. J Clin Oncol. 2012;30(Suppl 4):abstract 5.
20. Shen L, Li J, Xu J, et al. Bevacizumab plus capecitabine and cisplatin in Chinese patients with inoperable locally advanced or metastatic gastric or gastroesophageal junction cancer: randomized, double-blind, Phase III study (AVATAR study). Gastric Cancer. February 21, 2014.

21. Fong GH, Rossant J, Gertsentein M, Breitman ML. Role of the Flt-1 receptor tyrosine kinase in regualting the assembly of vascular endothelium. Nature. 1995;376(6535):66-70.

22. Spratlin JL, Cohen RB, Eadens M, et al. Phase I pharmacologic and biologic study of ramucirumab (IMC-1121B), a fully human immunoglobulin G1 monoclonal antibody targeting the vascular endothelial growth factor receptor-2. J Clin Oncol. 2010;28(5):780-787.

23. Fuchs C, Tomasek J, Yong CJ, et al. Ramucirumab monotherapy for previously treated advanced gastric or gastro-oesophageal junction adenocarcinoma (REGARD): an international, randomised, multicentre, placebo-controlled, Phase III trial. Lancet. 2014;383(9911): 31-39.

24. Ford H, Marshall A, Wadsley J, et al. Cougar-02: a randomized Phase III study of docetaxel versus active symptom control in advanced esophagogastric adenocarcinoma [abstract]. J Clin Oncol. 2013;31(Suppl 4): abstract LBA4.

25. Wilke H, Van Cutsem E, Oh SG, et al. RAINBOW: a global, Phase III, randomized, double-blind study of ramucirumab plus paclitaxel versus placebo plus paclitaxel in the treatment of metastatic gastroesophageal junction (GEJ) and gastric adenocarcinoma following disease progression on first-line platinum- and fluoropyrimidine-containing combination therapy rainbow IMCL CP12-0922 (I4T-IE-JVBE) [abstract]. J Clin Oncol. 2014;32(Suppl 3):abstract LBA7.

26. Van Cutsem E, de Haas S, Kang YK, et al. Bevacizumab in combination with chemotherapy as first-line therapy in advanced gastric cancer: a biomarker evaluation from the AVAGAST randomized Phase III trial. J Clin Oncol. 2012;30(17):2119-2127.

27. The Cancer Genome Atlas Research Network; Analysis Working Group: Dana-Farber Cancer Institue; Institute for Systems Biology, et al Comprehensive molecular characterization of gastric adenocarcinoma. Nature. Epub July 23, 2014.
Gastrointestinal Cancer: Targets and Therapy

\section{Publish your work in this journal}

Gastrointestinal Cancer: Targets and Therapy is an international, peer-reviewed, open access journal focusing on gastro-intestinal cancer research, identification of therapeutic targets and the optimal use of preventative and integrated treatment interventions to achieve improved outcomes, enhanced survival and quality of life for the

\section{Dovepress}

cancer patient. The manuscript management system is completely online and includes a very quick and fair peer-review system. Visit http://www.dovepress.com/testimonials.php to read real quotes from published authors 